International Journal of Pure and Applied Mathematics

Volume 99 No. 1 2015, 97-108

ISSN: 1311-8080 (printed version); ISSN: 1314-3395 (on-line version)

url: http://www.ijpam.eu

doi: http://dx.doi.org/10.12732/ijpam.v99i1.8

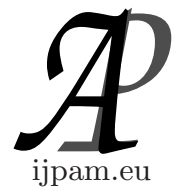

\title{
ORTHOGONAL GENERALIZED DERIVATIONS ON SEMIRINGS
}

\author{
N. Sugantha Meena ${ }^{1}$, M. Chandramouleeswaran ${ }^{2} \S$ \\ ${ }^{1}$ Department of Mathematics \\ Kamaraj College of Engineering and Technology \\ Virudhunagar, INDIA \\ ${ }^{2}$ Department of Mathematics \\ SBK College \\ Arupukottai, 626101, INDIA
}

\begin{abstract}
In [6] we discussed the notion of orthogonal derivations on semirings. In this paper, we introduce the notion of orthogonal generalized derivations on semirings and prove some results on semirpime semirings.
\end{abstract}

AMS Subject Classification: $16 \mathrm{Y} 30$

Key Words: semirings, semiprime semiring, generalized derivations, orthogonal derivations, orthogonal generalized derivations

\section{Introduction}

A semiring $(S,+, \cdot)$ is an algebraic structure such that $(S,+)$ and $(S, \cdot)$ are semigroups connected by ring like distributive laws. The study of semiring dates back to H.S.Vandiver[8]. In 1957, Posner proved two striking results on derivations on prime rings [5]. In [4], Jonathan Golan introduced the term derivation on semirings but nothing has been discussed on it. In [2],[3] Chandramouleeswaran and Thiruveni studied the notion of derivations on semirings in detail.

In [1], Bresar and Vukuman introduced the notion of orthogonality for a pair $\mathrm{d}, \mathrm{g}$ of derivations on a semiprime ring and they gave several necessary and sufficient conditions for $\mathrm{d}$ and $\mathrm{g}$ to be orthogonal. In [7], the author discussed the notion of orthogonal derivations and orthogonal generalized derivation on $\Gamma \mathrm{M}$ modules. Motivated by these works, in [6] we introduced the notion of orthogonal derivations on semirings. In this paper, we define and discuss the notion of orthogonal generalized derivation on semirings and prove some simple but elegant results on semiprime semirings. 
Definition 5. A semiring $S$ is said to be 2-torsion free if $2 a=0 \Longrightarrow$ $a=0, a \in S$.

Definition 6. A semiring $(S,+, \cdot)$ is said to be a semiring with zero, if it has an element 0 in $S$ such that

$$
x+0=0=0+x \text { and } x \cdot 0=0=0 \cdot x \quad \forall x \in S .
$$

Definition 7. An additive mapping $d: S \rightarrow S$ is called a derivation if

$$
d(x y)=d(x) y+x d(y) \forall x, y \in S .
$$

Definition 8. Let $(S,+, \cdot)$ be a semiring and let $d$ be a nontrival derivation on $S$. An additive mapping $D: S \rightarrow S$ is called a generalized derivation on $S$, associated with the derivation $d$, if

$$
D(x y)=D(x) y+x d(y) \quad \forall x, y \in S .
$$

Lemma 2.1. [6] Let $S$ be a 2- torsion free semiprime semiring, $a$ and $b$ the elements of $S$, then the following are equivalant.

1. $a s b=0$

2. $b s a=0$

3. $a s b+b s a=0$.

If one of these conditions are fulfilled then $a b=b a=0$

Lemma 2.2. [6] Let $S$ be a 2 torsion free semiprime semiring. Suppose that additive mappings $f$ and $g$ of a semiring $S$ into $S$ satisfying $f(x) s g(x)=$ $0, \forall x \in S$ then $f(x) s g(y)=0 \forall x, y \in S$.

Lemma 2.3. [6] Let $S$ be a 2 torsion free semiprime Semiring and let $d$ and $g$ be derivations of $S$ in to $S$. Derivations $d$ and $g$ are orthogonal iff $d(x) g(y)+g(x) d(y)=0 \forall x, y \in S$.

Lemma 2.4. [6] Let $S$ be a 2 torsion free semiprime semiring. Suppose $d$ and $g$ are derivations of $S$ in to $S$. Then $d$ and $g$ are orthogonal iff $d g=0$.

Lemma 2.5. [6] Let $S$ be a 2 torsion free semiprime Semiring.Suppose d and $g$ are derivations of $S$ in toS. Thend andg are orthogonal iff $d g+g d=0$.

Lemma 2.6. [6] Le $t S$ be a 2 torsionfree semiprime semiring. Supposed and $g$ are derivations of $S$ into $S$. Then $d$ and $g$ are orthogonal iff $d g$ is a derivation.

Notation Throughout this paper we shall assume that $\mathrm{S}$ is a 2-torsion free semiprime semiring with 0 and 1. 


\section{Orthogonal Generalized Derivation on Semirings}

In this section we introduce the notion of generalized orthogonal derivations and prove some results.

Definition 9. Let $S$ be a semiring. Let $d$ and $g$ be derivations on S. Let $D$ and $G$ be generalized derivations on $S$ associated with $d$ and $g$ respectively. $D$ and $G$ are said to be orthogonal generalized derivations if $D(x) S G(y)=$ $G(x) S D(y) \forall \mathrm{x}, \mathrm{y} \in \mathrm{S}$

Example 1. Let $S=\{0, a, b, c\}$.Define the operations + and . on $S$ as follows

\begin{tabular}{|c|c|c|c|c|}
\hline+ & 0 & $a$ & $b$ & $c$ \\
\hline 0 & 0 & $a$ & $b$ & $c$ \\
\hline$a$ & $a$ & $b$ & $c$ & $c$ \\
\hline$b$ & $b$ & $c$ & $c$ & $c$ \\
\hline$c$ & $c$ & $c$ & $c$ & $c$ \\
\hline
\end{tabular}

\begin{tabular}{|c|c|c|c|c|}
\hline$\cdot$ & 0 & $a$ & $b$ & $c$ \\
\hline 0 & 0 & 0 & 0 & 0 \\
\hline$a$ & 0 & $a$ & $b$ & $c$ \\
\hline$b$ & 0 & $b$ & $c$ & $c$ \\
\hline$c$ & 0 & $c$ & $c$ & $c$ \\
\hline
\end{tabular}

Then $(S,+, \cdot)$ is a semiring.

Define $d: S \rightarrow S$ such that $d(0)=0$ and $d(x)=c$ when $x=a, b, c$ $g: S \rightarrow S$ such that $g(0)=0, g(a)=b$ and $g(x)=c$ when $x=b, c$ clearly $\mathrm{d}$ and $\mathrm{g}$ are derivations on the semiring $\mathrm{S}$.

$D=d+b_{L}$ and $G=g+c_{L}$ where $b_{L}$ and $c_{L}$ denotes the left multiplication Let $\mathrm{S}_{1}=\mathrm{S} \oplus \mathrm{S}$.

Define $\mathrm{d}_{1}: \mathrm{S}_{1} \rightarrow \mathrm{S}_{1}$ by $\left.\mathrm{d}_{1}(x, y)\right)=(d(x), 0)$

$g_{1}: S_{1} \rightarrow S_{1}$ by $g_{1}(x, y)=(0, g(y))$,

$d_{1}$ and $g_{1}$ are orthogonal derivations.

Define $D_{1}: S_{1} \rightarrow \mathrm{S}_{1}$ by $D_{1}(x, y)=((D(x), 0)$

Define $G_{1}: S_{1} \rightarrow \mathrm{S}_{1}$ by $G_{1}(x, y)=(0, G(y))$

$D_{1}$ and $G_{1}$ are orthogonal generalized derivations with derivations $d_{1}$ and $g_{1}$

Theorem 3.1. Let $D$ and $G$ be orthogonal generalized derivations on $S$ associated with derivations $d, g$ respectively then the following relations hold

1. $D(x) G(y)=G(x) D(y)=0$. Hence $D(x) G(y)+G(x) D(y)=0 \forall x, y \in S$.

2. $d$ and $G$ are orthogonal and $d(x) G(y)=G(x) d(y)=0$

3. $g$ and $D$ are orthogonal and $g(x) D(y)=D(x) g(y)=0$

4. $d$ and $g$ are orthogonal derivations

Proof. 1) Since $D$ and $G$ are orthogonal $D(x) z G(y)=G(x) z D(y)=0$. 
By lemma 2.9 $D(x) G(y)=G(x) d(y)=0$.

Hence $D(x) G(y)+G(x) D(y)=0 \quad \forall x, y \in S$.

2) From (1) $D(x) G(y)=0$.

Replacing $x$ by $z x, z \in S$ we get

$$
\begin{aligned}
0 & =D(z x) G(y) \\
& =D(z) x G(y)+z d(x) G(y) \\
& =z d(x) G(y) \quad(\text { Since } D \text { and } G \text { are orthogonal })
\end{aligned}
$$

That is $z d(x) G(y)=0$

premultiplying by $d(x) G(y)$ we get

$(d(x) G(y)) z(d(x) G(y))=0, \forall z \in S$

That is $(d(x) G(y)) S(d(x) G(y))=0 \forall x, y, z \in S$

Since $\mathrm{S}$ is semiprime,

$$
d(x) G(y)=0
$$

Replacing $x$ by $x z$ in 3.1 we have

$$
\begin{aligned}
0 & =d(x z) G(y) \\
& =d(x) z G(y)+x d(z) G(y) \\
& =d(x) z G(y) \text { By } 3.1
\end{aligned}
$$

That is

$$
d(x) z G(y)=0
$$

From result (1) $0=G(x) D(y)$

Replacing $y$ by $z y, z \in S$, we get

$$
\begin{aligned}
0= & G(x) D(z y) \\
= & G(x) D(z) y+G(x) z d(y) \\
= & G(x) z d(y) \quad \text { By result }(1) \\
& G(x) z d(y)=0
\end{aligned}
$$

By lemma 2.9 we have $G(x) d(y)=0$.

Also from 3.2 and 3.3

$$
d(x) z G(y)=0=G(x) z d(y) \forall x, y, z \in S
$$


Hence $d$ and $G$ are orthogonal.

Analogously we can prove result (3).

(4) Suppose $0=D(x) G(y)$.

Replacing $x$ by $x z$ and $y$ by $y u$ we have

$$
\begin{aligned}
0 & =D(x z) G(y u) \\
& =(D(x) z+x d(z))(G(y) u+y g(u)) \\
& =D(x) z G(y) u+D(x)(z y) g(u)+x d(z) G(y) u+x d(z) y g(u) \\
& =x d(z) y g(u) \text { by result }(2) \text { and }(3)
\end{aligned}
$$

That is $x d(z) y g(u)=0$.

Pre multiplying by $d(z) y g(u)$ we have

$$
(d(z) y g(u)) x(d(z) y g(u))=0 \forall u, x, y, z \in S .
$$

That is,

$$
(d(z) y g(u)) S(d(z) y g(u))=0 \forall u, x, y, z \in S .
$$

By semiprimeness of $S$, we have

$$
d(z) y g(u)=0 \forall u, y, z \in S .
$$

Similarly, from $G(x) D(y)=0$ we can prove that $g(z) y d(u)=0 \forall u, y, z \in S$.

This completes the proof that $d$ and $g$ are orthogonal.

Lemma 3.1. Let $S$ be a 2- torsion semiprime semiring and let $D$ be a generalized derivation on $S$ associated with a derivation $d$. If $D(x) D(y)=$ $0 \forall x, y \in S$, then $D=d=0$.

Proof. Replacing $y$ by $y z$ in the given condition $D(x) D(y)=0 \forall x, y \in S$ we get

$$
\begin{aligned}
0 & =D(x) D(y z) \\
& =D(x)((D(y) z+y d(z)) \\
& =D(x) D(y) z+D(x) y d(z) \\
& =D(x) y d(z)
\end{aligned}
$$

By lemma 2.9,

$$
D(x) d(z)=d(z) D(x)=0 \forall x, z \in S
$$

Replacing $x$ by $x z$ in $d(z) D(x)=0$ we get

$$
0=d(z) D(x z)
$$




$$
\begin{aligned}
& =d(z)((D(x) z+x d(z)) \\
& =d(z) D(x) z+d(z) x d(z) \\
& =d(z) y d(z) \text { By } 3.4
\end{aligned}
$$

Since $S$ is semiprime $d(z)=0, \forall z \in S$

Replace $x$ by $y x$ in $D(x) D(y)=0$ we get

$$
\begin{aligned}
0 & =D(y x) D(y) \\
& =(D(y) x+y d(x)) D(y) \\
& =D(y) x D(y)+y d(x) D(y) \\
& =D(y) x D(y) \quad B y 3.4
\end{aligned}
$$

Since $S$ is semiprime, $D(y)=0, \forall y \in S$

Hence $D=d=0$.

Lemma 3.2. Let $D$ and $G$ be generalized derivations on a semiring $S$ with derivations $d$ and $g$ respectively. If the following relations hold

(i) $D(x) G(y)+G(x) D(y)=0$

(ii) $d(x) G(y)+g(x) D(y)=0$

then $D$ and $G$ are orthogonal.

Proof. Replacing $x$ by $x z$ in (i) we have

$$
\begin{aligned}
0 & =D(x z) G(y)+G(x z) D(y) \\
& =(D(x) z+x d(z)) G(y)+(G(x) z+x g(z)) D(y) \\
& =D(x) z G(y)+x d(z) G(y)+G(x) z D(y)+x g(z) D(y) \\
& =D(x) z G(y)+x(d(z) G(y)+g(z) D(y))+G(x) z D(y) \\
& =D(x) z G(y)+G(x) z D(y) B y(i i)
\end{aligned}
$$

That is

$$
D(x) z G(y)+G(x) z D(y)=0, \forall x, y, z \in S
$$

Replacing $y$ by $x$ we have

$D(x) z G(x)+G(x) z D(x)=0$

by lemma $2.9 D(x) z G(x)=0, \forall x, z \in S$

By lemma 2.10 $D(x) z G(y)=0$

similarly replacing $x$ by $y$ in 3.5 we have $G(y) z D(y)=0$

by lemma $2.10 G(y) z D(x)=0 \forall x, y, z \in S$.

$\mathrm{D}$ and $\mathrm{G}$ are orthogonal 
Lemma 3.3. Let $S$ be a 2 - torsion free semiprime Semiring. Suppose $D$ and $G$ are generalized derivations on $S$ with derivations $d$ and $g$ respectively such that $D(x) G(y)=d(x) G(y)=0 \forall x, y \in S$, then $D$ and $G$ are orthogonal.

Proof. Replacing $x$ by $x z$ in $D(x) G(y)=0$, we get

$$
\begin{aligned}
0 & =D(x z) G(y) \\
& =(D(x) z+x d(z)) G(y) \\
& =D(x) z G(y)+x d(z) G(y) \\
& =D(x) z G(y)
\end{aligned}
$$

That is $D(x) z G(y)=0, \forall x, y, z \in S$.

By lemma $2.9 G(y) z D(x)=0$.

Replacing $y$ by $x$ we get $G(x) z D(x)=0$.

By lemma $2.10 G(x) z D(y)=0, \forall x, y, z \in S$.

Thus we have proved that $D$ and $G$ are orthogonal.

Lemma 3.4. Let $S$ be a 2 - torsion free semiprime Semiring. Suppose $D$ and $G$ are generalized derivations on $S$ with derivations $d$ and $g$ respectively such that $D(x) G(y)=0, d G=d g=0$. Then $D$ and $G$ are orthogonal.

Proof. Suppose $d g=0$. By lemma 2.12, $d$ and $g$ are orthogonal.

Therefore $d(x) z g(y)=0$ and $g(x) z d(y)=0 \forall x, y, z \in S$.

By lemma 2.9

$$
d(x) g(y)=0=g(x) d(y)
$$

Suppose $d G=0$. That is, $d G(x)=0 \forall x \in S$.

Replacing $x$ by $x y$, we get

$$
\begin{aligned}
0=d G(x y) & =d(G(x y) \\
& =d(G(x) y+x g(y)) \\
& =d G(x) y+G(x) d(y)+d(x) g(y)+x d g(y) \\
& =G(x) d(y) \quad B y 3.6
\end{aligned}
$$

Replacing $x$ by $x z$, we have $G(x z) d(y)=0$.

That is, $G(x) z d(y)+x g(z) d(y)=0$.

By $3.6 G(x) z d(y)=0 \forall x, y, z \in S$.

By lemma $2.9 d(y) z G(x)=0$ and $d(y) G(x)=0$.

By lemma 3.6 $D$ and $G$ are orthogonal.

Lemma 3.5. Let $S$ be a 2-torsion free semiprime Semiring. Suppose $D$ and $G$ are Generalized derivations on $S$ associated with derivations $d$ and $g$ 
respectively such that $D G$ is a generalized derivation on $S$ with derivation $d g$ and $D(x) G(y)=0 \forall x, y \in S$, then $D$ and $G$ are orthogonal.

Proof.

$$
\begin{aligned}
D G(x y) & =D(G(x) y+x g(y)) \\
& =D G(x) y+G(x) d(y)+D(x) g(y)+x d g(y)
\end{aligned}
$$

But $D G(x y)=D G(x) y+x d g(y)$. Comparing

$$
G(x) d(y)+D(x) g(y)=0
$$

Replacing $y$ by $y z$ in $D(x) G(y)=0$ we have $D(x) y g(z)=0$.

By lemma $2.9 g(z) D(x)=0$.

Replacing $z$ by $y z$, we have

$$
\begin{aligned}
0 & =g(y z) D(x) \\
& =g(y) z D(x)+y g(z) D(x) \\
& =g(y) z D(x)
\end{aligned}
$$

By lemma $2.9 D(x) g(y)=0 \quad \forall x, y \in S$.

Using in $3.7 G(x) d(y)=0$

Replacing $y$ by $y z$ we have $G(x) d(y z)=0$.

$G(x) d(y) z+G(x) y d(z)=0$

using $G(x) d(y)=0$ we have $G(x) y d(z)=0 \forall x, y, z \in S$.

By lemma $3.3 d(z) G(x)=0 \forall x, z \in S$.

By lemma 3.6 $D$ and $G$ are orthogonal.

\section{Products of Generalized Derivation on Semirings}

In this section we prove a necessary and sufficient condition for the product of two generalized derivations to be a generalized derivation in terms of the orthogonal derivations.

Lemma 4.1. Let $D$ and $G$ be generalized derivations on $S$ associated with derivations $d$ and $g$ respectively. Then $D G$ is a generalized derivation associated with the derivation $d g$ if and only if $D$ and $g$ and $G$ and $d$ are orthogonal derivations.

Proof. Assume $D G$ is a generalized derivation with derivation $d g$.

By lemma $2.14 d$ and $g$ are orthogonal derivations. 
Also by equation 3.7, $G(x) d(y)+D(x) g(y)=0$.

Replacing $y$ by $y z$ we have

$$
\begin{aligned}
0 & =G(x) d(y z)+D(x) g(y z) \\
& =G(x) d(y) z+G(x) y d(z)+D(x) g(y) z+D(x) y g(z) \\
& =(G(x) d(y)+D(x) g(y)) z+G(x) y d(z)+D(x) y g(z) B y 3.7
\end{aligned}
$$

That is,

$$
G(x) y d(z)+D(x) y g(z)=0 \forall x, y \in S .
$$

Replacing $x$ by $x g(z)$ we have

$$
\begin{aligned}
0 & =G(x g(z)) y d(z)+D(x g(z)) y g(z) \\
& =G(x) g(z) y d(z)+x g(g(z)) y d(z)+D(x) g(z) y g(z)+x d(g(z)) y g(z) \\
& =D(x)(g(z) y) g(z)=0 \forall x, y, z \in S
\end{aligned}
$$

By Lemma $2.9 D(x) g(z)=0$.

Replacing $z$ by $y z$ we get and using the above condition, we have $D(x) y g(z)=$ 0.

Now, equation 4.1 implies that $G(x) y d(z)=0$. By lemma 2.9

$$
d(z) G(x)=0
$$

Replacing $z$ by $x y$ and $x$ by $z y$, we get

$$
\begin{aligned}
0 & =d(x y) G(z y) \\
& =(d(x) y+x d(y))(G(z) y+z g(y)) \\
& =d(x) y G(z) y+d(x)(y z) g(y)+x d(y) G(z) y+x d(y) z g(y) \\
& =d(x) y G(z) y \text { By equation } 4.2 \text { and orthogonality of } d \text { and } g
\end{aligned}
$$

Postmultiplying by $d(x) y G(z)$ we have $(d(x) y G(z)) y(d(x) y G(z))=0$.

Since $S$ is semiprime $d(x) y G(z)=0 \forall x, y, z \in S$.

Therefore $G$ and $d$ are orthogonal.

Analogously we can prove that $D$ and $g$ are orthogonal.

Conversely assume that both the pairs $D$ and $g$ and $G$ and $d$ are orthogonal.

Now by the orthogonality of $\mathrm{D}$ and $\mathrm{g}$ we have

$D(x) y g(z)=0=g(x) y D(z) \forall x, y, z \in S$.

By lemma $2.9 D(x) g(z)=0 \forall x, z \in S$.

Replacing $x$ by $r x$ in $D(x) y g(z)=0$

$$
0=D(r x) y g(z)
$$




$$
\begin{aligned}
& =D(r) x g(z)+r d(x) y g(z) \\
& =\operatorname{rd}(x) y g(z)
\end{aligned}
$$

Premultiplying by $d(x) y g(z)$ we have $(d(x) y g(z)) r(d(x) y g(z))=0$.

By semiprimeness of $S, d(x) y g(z)=0 \forall x, y, z \in S$.

By the orthogonality of $G$ and $d$ we have

$G(x) y d(z)=0=d(x) y G(z) \forall x, y, z \in S$.

By lemma $2.9 G(x) d(z)=0 \forall x, z \in S$.

Replacing $x$ by $z x$ in $G(x) y d(z)=0$ we have $g(x) y d(z)=0 \forall x, y, z \in S$.

Then $d$ and $g$ are orthogonal derivations.

By lemma $2.14 d g$ is a derivation

Using $d(x) y g(z)=0, D(x) g(y)=0, G(x) d(y)=0$ in

$D G(x y)=D G(x) y+G(x) d(y)+D(x) g(y)+x d g(y)$

we have $D G(x y)=D g(x) y+x d g(y)$.

Thus $D G$ is a generalized derivation with derivation $d g$.

Corollary 4.1. Let $D$ and $G$ be generalized derivations on $S$ associated with derivations $d$ and $g$, then $G D$ is generalized derivation on $S$ with derivation gd iff $D$ and $g, G$ and $d$ are orthogonal.

Lemma 4.2. Let $D$ be a generalized derivation on $S$ associated with derivation $d$. If $D^{2}$ is a generalized derivation with derivation $d^{2}$ then $d=0$.

Proof. Since $d^{2}$ is a derivation, $d$ and $d$ orthogonal by lemma 2.14.

Hence $d(x) y d(z)=0 \forall x, y, z \in S$. Replacing $z$ by $x, d(x) y d(x)=0$ Since $S$ is semiprime $d(x)=0 \forall x \in S$.

Lemma 4.3. Let $S$ be a 2 - torsion free prime semiring. If $D$ and $G$ are generalized derivations on $S$ associated with derivations $d$ and $g$ respectively such that $D$ and $g, G$ and $d$ are orthogonal, then $D=d=0$ or $G=g=0$.

Proof. Since $D$ and $g$ are orthogonal we have

$$
D(x) y g(z)=0 \text { and } g(x) y D(z)=0 \forall x, y, z \in S \text {. }
$$

Since $G$ and $d$ are orthogonal we have

$$
G(x) y d(z)=0 \text { and } d(x) y G(z)=0 \forall x, y, z \in S \text {. }
$$

Since $S$ is prime and $D(x) y g(z)=0$ we have $D(x)=0$ or $g(z)=0 \forall x, z \in S$. From $d(x) y G(z)=0$ we have $d(x)=0$ or $G(z)=0$

Therefore $D=d=0$ or $G=g=0$ 
Lemma 4.4. If $D$ and $G$ are orthogonal generalized derivations on $S$ associated with derivations $d$ and $g$ respectively, then $d G=G d=0$ and $g D=$ $D g=0, D G=G D=0$.

Proof. By result (2),(4) of theorem $3.3 d$ and $G, d$ and $g$ are orthogonal.

Hence $d(x) z G(y)=0$ and $d(x) z g(y)=0, d(x) g(y)=0 \forall x, y, z \in S$.

$$
\begin{aligned}
0 & =G(d(x) z G(y)) \\
& =G d(x) z G(y)+d(x) g(z G(y)) \\
& =G d(x) z G(y)+d(x) g(z) G(y)+d(x) z g(G(y)) \\
& =G d(x) z G(y) \forall x, y, z \in S
\end{aligned}
$$

That is $G(d(x)) z G(y)=0 \forall x, y, z \in S$

Replacing $y$ by $d(x)$ we have $G(d(x)) z G(d(x))=0$

Since $S$ is semiprime, $G d=0$

Similarly from $d(G(x) z d(y))=0, D(g(x) z D(y))=0, g(D(x) z g(y))=0$ and $G(D(x) z G(y))=0$

we have $d G=D g=g D=D G=G D=0$.

\section{References}

[1] M. Bresar, J. Vukuman, Orthogonal derivation and extension of a theorem of Posner, Radovi Matematicki, 5 (1989), 237.

[2] M. Chandramouleeswaran, V. Thiruveni, On derivations of semirings, Advances in Algebra, 3 (2010), 123-131.

[3] M. Chandramouleeswarn, V. Thiruveni, A note on $\alpha$ derivations in semirings, International Journal of Pure and Applied Science and Technology (2011), 71-77.

[4] Jonathan S. Golan, Semirings and their applications, Kluwar Academic Press (1969).

[5] E.C. Posner, Derivation in Prime Rings, American Math. Soc., 8 (1957), 1093-1100.

[6] Meena N. Sugantha, M. Chandramouleeswaran, Orthogonal derivations on semirings, Submitted to International Journal of Contemporary Mathematical Sciences. 
[7] Salah Mehdi Salih, Orthogonal derivations and generalized orthogonal derivations on $\gamma \mathrm{M}$ modules, Iraqi Journal of Science, 54, No. 3 (2013), 658-665.

[8] H.S. Vandiver, Note on a simple type of algebra in which the cancellation law of addition does not hold, Bull. Amer. Math. Soc., 40 (1934), 916-920. 